\title{
Efficient Lattice-Image Detection of Icosahedral Twins
}

\author{
P. Fraundorf ${ }^{1,2}$ and Chris Bishop ${ }^{2,3}$ \\ 1. Center for NanoScience, University of Missouri StL, Saint Louis MO 63121, USA \\ 2. Physics \& Astronomy, University of Missouri StL, Saint Louis MO 63121, USA \\ 3. present address: Physics Department, University of Tennessee, Knoxville TN, USA
}

Icosahedral clustering is characteristic of the many common "face-centered-cubic metals" on the nanoscale, either in liquid [1] and/or in solid form. As the surface-to-volume ratio of a growing solid icosahedral-cluster decreases, however, bulk-atoms typically re-arrange themselves into their space-filling fcc form, turning the cluster in to a coordinated-set of paired-crystal icosahedral-twins.

These crystal-pairs "light up" like opposing pie-slices in both analog and digital darkfield images [2]. Single pairs (from any active reflection) oft have the appearance of "bowties" e.g. from the (200) reflection parallel to the bowtie axis or from the (220) reflection perpendicular to that axis. Double-pairs have the appearance of "butterflies" e.g. from the (111) reflection perpendicular to the interface between adjacent tetrahedra.

Analysis of the visibility of lattice fringes [3,4] from a subset of the particles in a 10-crystal icosahedral twin indicates most randomly-oriented particles in the $10 \mathrm{~nm}$ diameter range will show lattice fringes in a bow-tie and/or butterfly pattern. These characteristic shapes, in turn, are likely to show up in a frequency-space tiled digital-darkfield tableau [5]. The detectable fraction will be even closer to $100 \%$ for smaller particles, and may still be above $2 / 3$ for particles as large as $50 \mathrm{~nm}$ in diameter (to the extent that lattice contrast is not washed out by multiple-scattering).

The geometry of great-circle band intersection-areas is messy, and the lattice-fringe visibility map of an icosahedral-twin is more complicated still. We therefore developed 2D-projection "paintmethods" for estimating the fraction of randomly-oriented icosahedral-twin particles of a given thickness $\mathrm{t} / \Gamma$, where $\Gamma$ is a shape-transform signal/noise correction-factor on the order of 1 . Calibration of these methods, and $\Gamma$ 's relationship particle-shape \& noise, are discussed in a separate papers here on (i) geometry vs. paint models of fcc lattice-fringe visibility and (ii) lattice-image determination of nanoparticle fraction-crystalline.

The color stereo-projections in Fig. 2 show how massive amounts of band overlap (as crystal size shrinks) can be quantified for three separate band-spacings using RGB color images, in this case (111), (200) and (220) fringes when means lattice fringe contrast to about 2.3, 2, and 1.4A respectively. Fig. 3 zooms in on these bands in the ico-twin's asymmetric unit, whose low-angle band-overlaps makes analytical calculation of areas prohibitive.

Fig. 4 plots estimates for the fraction of randomly-oriented ico-twin clusters that will show detectable (111), (200) or (220) lattice fringes (and hence triangle-sections in a digital-darkfield tableau) as a function of size, inferred from $2 \pi$-steradian $512 \times 512$ stereographic paint-maps. The top (red) points and fit-curve were obtained (CB) from one asymmetric unit of a monochrome map, while the bottom (blue) points and fit-curve were obtained (PF) from all pixels of a 3-band color-overlay map, both corrected for projection-distortion. 
Awaiting experimental data on $\Gamma$ for a given scope/specimen combination, the results suggest that this method will detect icosahedral-twinning in a sizeable fraction of randomly-oriented clusters less than $20 \mathrm{~nm}$ in size. Reliable detection of lattice-fringes, at least down to (200), may be a pre-requisite.

\section{References:}

[1] K. F. Kelton et al. (2003) Phys. Rev. Lett. 90, 195504.

[2] P. Fraundorf (2004) Digital Darkfield Decompositions (arXiv:cond-mat/0403017).

[3] P. Fraundorf et al. (2005) Journal of Applied Physics 98:114308.

[4] P. Wang et al. (2006), Ultramicroscopy 106:277-283.

[5] P. Fraundorf et al. (2006) Microscopy and Microanalysis 12:S2: 1010-1011.
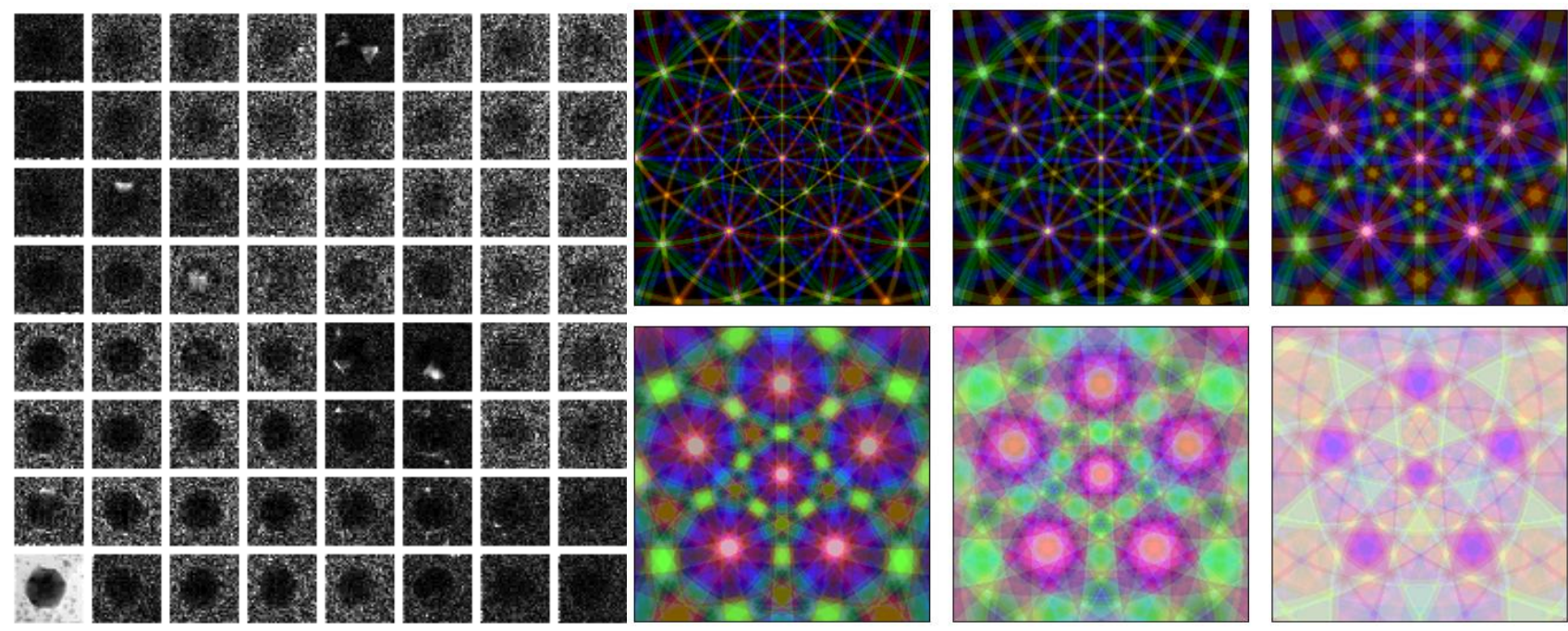

Fig 1 (left): Part of a $32 \times 32$ digital darkfield array from a $1024 \times 1024$ randomly-oriented icotwin.

Fig 2 (right): icotwin $300 \mathrm{kV}$ red-blue-green visibility-maps for $\mathrm{t} / \Gamma=\{\{160,80,40\},\{20,10,5\}\} \AA$.

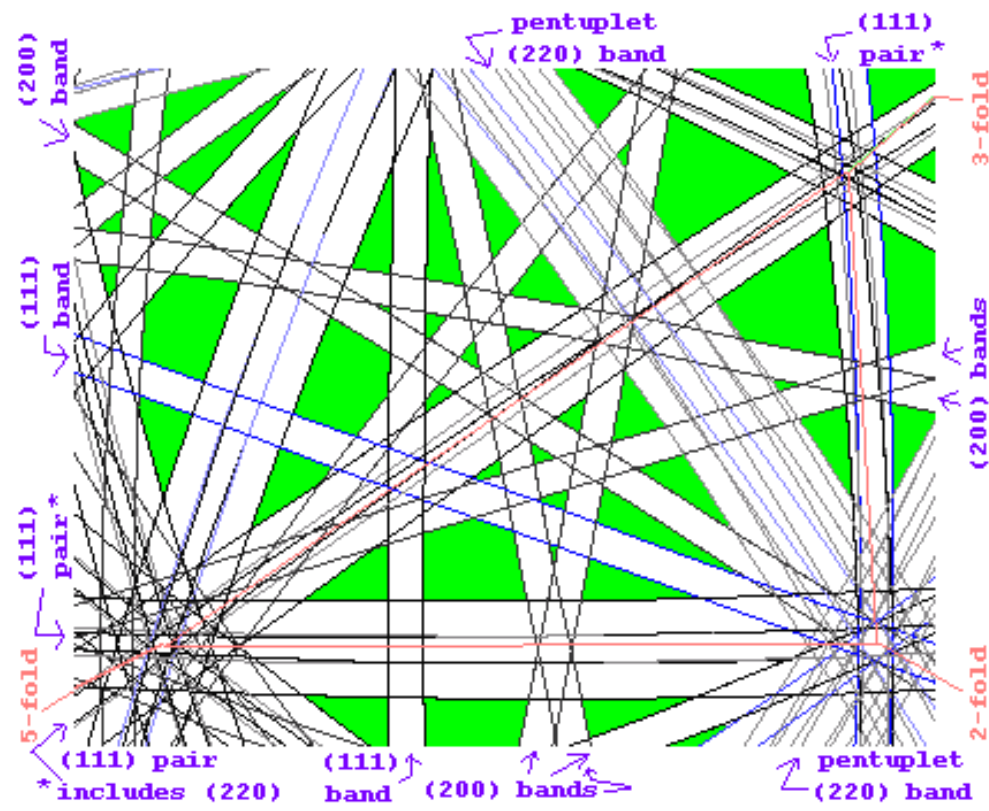

Two separate paint-model estimates

Fig. 3 (left): Icotwin asymmetric-unit (5-fold lower-left, 3-fold upper-right, 2-fold bottom right).

Fig. 4 (rght): Asymmetric-unit (red) \& color-overlay (blue) estimates: orientation-fraction detectable.. 